\title{
Parameterized Flight Mission for Secondary Power Requirement Estimations of Commercial Transport Aircraft
}

\author{
Thomas Lampl*, Sandra Muschkorgel ${ }^{\dagger}$, and Mirko Hornung \\ Technical University of Munich, Faculty of Mechanical Engineering, \\ Garching, 85748, Germany
}

\begin{abstract}
The trend towards More-Electric Aircraft (MEA) and the introduction of new system technologies lead to considerable changes at the system level of commercial transport aircraft. Because the number of systems and power requirements are increasing, the consideration and integration of aircraft systems in early aircraft design phases is important. The objective of this contribution is to develop a characteristic flight mission with modelled aircraft systems to estimate the secondary power requirements. Therefore, a parameterized reference mission with typical altitude and speed profile of commercial transport aircraft is defined. Moreover, two baseline aircraft representing a Medium-Range (MR) and a Long-Range (LR) configuration respectively, are defined. On basis of aircraft-level functions, simplified system models of the Environmental Control System (ECS), the Ice Protection System (IPS) and the Flight Control System (FCS) are derived. Whereas the power requirement estimations of other aircraft systems, e.g. galleys, avionics, and fuel systems, are mainly based on data from literature. The parametrized mission and system models are implemented into a tool and merged for calculations and analysis. This approach enables the aircraft-level analysis and assessment of system technologies and concepts in early aircraft design phases, regarding secondary power requirements.
\end{abstract}

\section{Nomenclature}

$\begin{array}{ll}A C P & =\text { Air Conditioning Pack } \\ C A C & =\text { Cabin Air Compressor } \\ E C S & =\text { Environmental Control System } \\ E I P S & =\text { Engine Ice Protection System } \\ F C S & =\text { Flight Control System } \\ I P S & =\text { Ice Protection System } \\ M E A & =\text { More-Electric Aircraft } \\ M I S & =\text { Miscellaneous Systems } \\ P C U & =\text { Power Control Unit } \\ W I P S & =\text { Wing Ice Protection System }\end{array}$

\section{Introduction}

A IRCRAFT systems play an important role for performance, efficiency and safety of commercial transport aircraft. With the introduction of electronic and computer controlled systems (e.g. fly-by-wire), aircraft systems have undergone many changes as well as more and more new aircraft systems and advanced systems technologies are introduced. Furthermore, the evolutionary application of enabling electrical and electronic technologies leads to substitution of some hydraulic and pneumatic systems.

In 1985, Tagge et al. ${ }^{1}$ and Hoffmann et al. ${ }^{2}$ investigated the idea of all-electric systems and predicted efficiency and weight improvements for advanced electric aircraft systems. Almost thirty years later, the latest introduced commercial transport aircraft - the Boeing 787, the Airbus A380 and A350 - show a notable increase in rated electrical generator power, see Fig. 1. The biggest leap were made with the 787 due to its bleed-less system architecture, with

\footnotetext{
* Research Assistant, Institute of Aircraft Design, lampl@tum.de, AIAA Student Member

${ }^{\dagger}$ Graduate Student, Institute of Aircraft Design, s.muschkorgel@tum.de

* Professor, Head of Institute of Aircraft Design, mirko.hornung@tum.de, AIAA Senior Member
} 
fully electrical Environmental Control System (ECS) and Ice Protection System (IPS) ${ }^{3}$. Smaller changes were made with the A380 and A350, as they still have a bleed-air system on board to supply the ECS and IPS. Nevertheless, new electrical technologies and concepts were introduced ${ }^{4}$. These aircraft can be defined as the first generation of MoreElectric Aircraft (MEA) in commercial transport aviation.

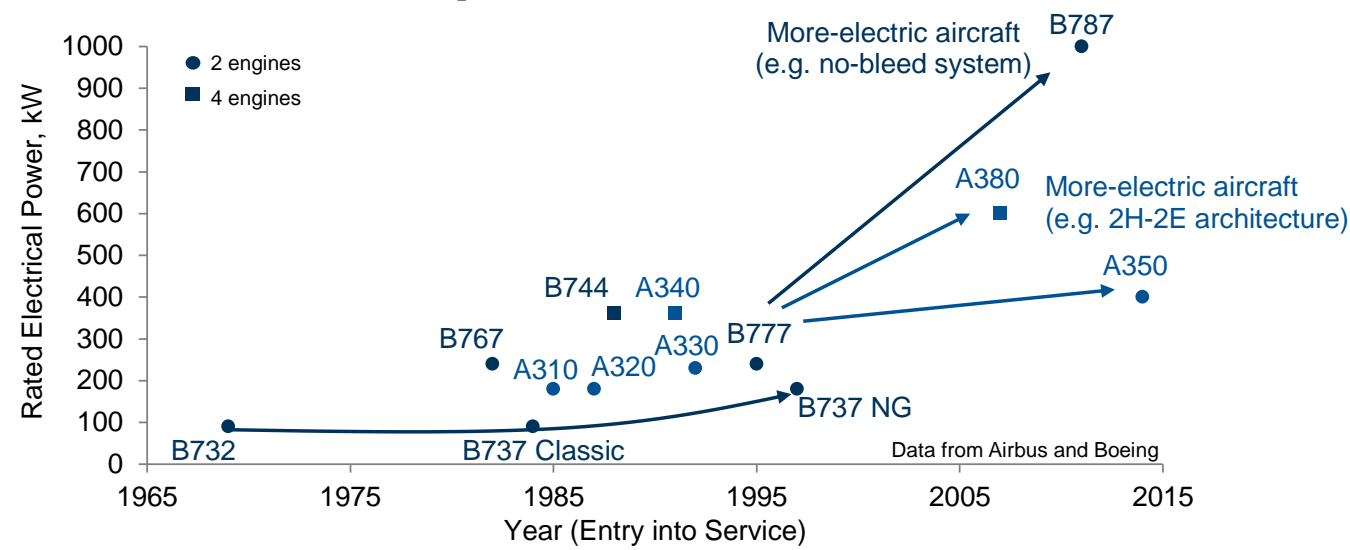

Figure 1. Evolution of electrical power of commercial transport aircraft (Airbus and Boeing).

On the aircraft level, the main impact of aircraft systems can be described by system masses and engine power offtakes required for secondary power. To enable a successful integration of advanced or new system technologies, an early consideration and integration of aircraft systems in preliminary aircraft design, is becoming more and more important ${ }^{5,6}$.

The objective of this contribution is to develop a representative flight mission with modelled aircraft systems for secondary power requirement estimations of commercial transport aircraft. Therefore, parameterized baseline aircraft and parameterized mission profiles are defined. For the main aircraft systems - the ECS, IPS and the Flight Control System (FCS) - simplified system models are developed. The power requirements of additional aircraft systems are estimated by semi-empirical approaches or data from literature review. Finally, the defined reference mission and aircraft system models are integrated into a calculation tool in MATLAB ${ }^{\circledR}$. The resulting tool enables the aircraftlevel analysis and assessment of conventional and new aircraft system technologies or architectures, regarding secondary power requirements.

\section{Baseline Aircraft and Mission Definition}

Flight missions of commercial transport aircraft are characterized by specific performance data (e.g. altitude and speed), aircraft-specific data (e.g. configuration and masses), official regulations and environmental conditions (e.g. atmosphere $)^{7}$. Therefore, two representing baseline aircraft and a parameterized mission with typical altitude and speed profile are defined.

\section{A. Baseline Aircraft Configuration}

Commercial transport aircraft are typically divided in Short-Range (SR), Medium-Range (MR) and Long-Range (LR) categories. As no clear definition or standard for categorization could be found in literature, two baseline aircraft configurations are defined to represent most of all commercial transport aircraft, see Table 1 .

Table 1. Main parameters of the baseline aircraft configurations.

\begin{tabular}{|c|c|c|c|c|c|c|c|}
\hline Parameter & MR & LR & Unit & Parameter & MR & LR & Unit \\
\hline General & & & & Wing & & & \\
\hline Crew ${ }^{a}$ & $2 / 4$ & $2 / 10$ & - & Span & 34.8 & 63.9 & $m$ \\
\hline Capacity $^{b}$ & 189/184/162 & $550 / 442 / 370$ & - & Area (ref.) & 124 & 440 & $m$ \\
\hline Length & 38.5 & 73.8 & $m$ & Sweep & 25 & 33 & $\circ$ \\
\hline Height & 12.2 & 17.8 & $m$ & Aspect ratio & 9.6 & 8.8 & - \\
\hline Mass & & & & Engine (2) & & & \\
\hline MTOM & 76390 & 329770 & $\mathrm{~kg}$ & Number & 2 & 2 & - \\
\hline$M L M$ & 65220 & 242150 & $\mathrm{~kg}$ & Thrust & 130 & 484 & $k N$ \\
\hline
\end{tabular}

American Institute of Aeronautics and Astronautics 
The first baseline aircraft represents a typical MR transport aircraft, comparable to a Boeing 737-800 and an Airbus A320-200. The second baseline aircraft represents a typical LR category aircraft, comparable to a Boeing 777-300ER or an Airbus A350-1000. Both baseline aircraft are twin-engine configurations.

\section{B. Mission Altitude and Speed Profile}

According to $\mathrm{Simsic}^{8}$, the mission profile can be described by an altitude and speed profile as a function of time. Furthermore, the speed $V$ of the aircraft is defined as function of the altitude $h$. In this study, it is assumed that the Indicated Air Speed (IAS) is equal to the Calibrated Air Speed (CAS). To get the True Air Speed (TAS), the compressibility of the air and the air density are considered. The TAS constitutes the basis for all calculations in context with the Mach number, see Eq. 1.

$$
V_{T A S}=M a \cdot \sqrt{\gamma \cdot R_{\text {spec }} \cdot T_{\infty}}
$$

The parameter $\gamma$ represents the heat capacity ratio of the air and $R_{\text {spec }}$ stands for the specific gas constant of air. The temperature $T_{\infty}$ describes the ambient air temperature. For simplification, no wind conditions are assumed, so that the ground speed is equal to the TAS. In low altitudes, the speed is typically given by the CAS. According to Nuic ${ }^{9}$, the conversion from CAS to TAS can be done using Eq. 2. Both, the air density $\rho(h)$ and air pressure $p(h)$ are dependent from the flight altitude $h$. The parameters $\rho_{0, I S A}$ and $p_{0, I S A}$ represents the ISA conditions on Mean Sea Level (MSL).

$$
V_{T A S}(h)=\sqrt{\frac{2 \gamma}{\gamma-1} \cdot \frac{p(h)}{\rho(h)}\left\{\left[1+\frac{p_{0, I S A}}{p(h)}\left(1+\frac{\gamma-1}{2 \gamma} \cdot \frac{\rho_{0, I S A}}{p_{0, I S A}} \cdot V_{C A S}^{2}\right)^{\frac{\gamma}{\gamma-1}}-1\right]^{\frac{\gamma-1}{\gamma}}-1\right\}}
$$

A typical flight mission of commercial transport aircraft can be divided in following main phases: Taxi-out, Takeoff, Climb, Cruise, Descent, Approach, Landing, and Taxi-in. The flight phase loiter for holding is optional and not considered within this study. Figure 2 shows the defined phases and altitudes of the flight mission. The corresponding parameters for the defined baseline aircraft (MR/LR), are given in Table 3 in the appendix.

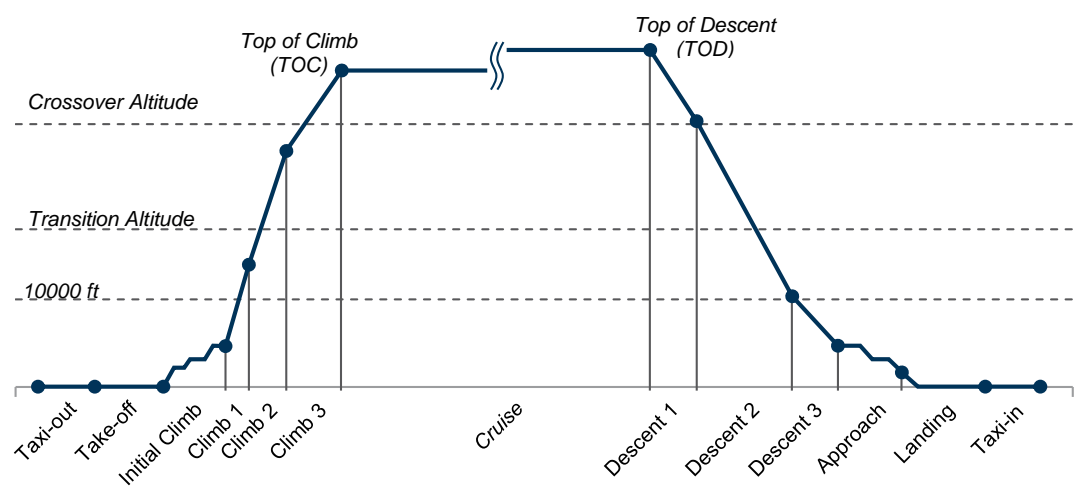

Figure 2. Mission phases and altitude profile schematics

The Taxi-out phase begins with the push-back and ends with the positioning of the aircraft on the runway. At standard conditions, the taxi speed is between $15-20 \mathrm{kt}$. The time accounting for this phase is 11 minutes *. The Takeoff phase starts with the beginning of acceleration on the runway and ends with reaching $35 \mathrm{ft}$ over ground (obstacle height). Furthermore, it is assumed that the take-off speed is equal to the speed for the beginning of the climb ( $\left.V_{2}\right)$.

The Climb phase comprises the whole climbing process until reaching cruise altitude. It is divided in Initial Climb and Climb 1, 2, and 3, that differ in the rate of climb, taken from the Aircraft Performance Database ${ }^{10}$ of Eurocontrol.

\footnotetext{
* The taxi-in and taxi-out times are based on averaged data for the period summer/winter 2012-2013 of the most relevant airports with minimum 500 flights in this period (Data from www.eurocontrol.int)
} 
According to Gillet et al. ${ }^{7}$, three phases of acceleration are made during Initial Climb, where the speed $\mathrm{V}_{2}$ increases by $10 \mathrm{kt}, 30 \mathrm{kt}$ and $60 \mathrm{kt}(\mathrm{CAS})$. The next acceleration is attempted after reaching $10000 \mathrm{ft}$. This altitude limits the maximum allowed CAS of $250 \mathrm{kt}$ (CAS) under FL100. After reaching the transition altitude at $18000 \mathrm{ft}$, the TAS and Mach number are continuously increased until Top of Climb (TOC) altitude and cruise speed (Ma) are reached.

The Cruise represents the largest phase of the flight mission, regarding to time and range. Standard cruise altitudes for the baseline aircraft are $40000 \mathrm{ft}(\mathrm{MR})$ and $42000 \mathrm{ft}$ (LR). The maximum cruise speed is limited by the maximum operating Mach number $M a_{M O}$. According to Rustenberg et al. ${ }^{11}$ and Tipps et al. ${ }^{12}$, typical $M a_{M O}$ for the baseline aircraft are $0.81(\mathrm{MR})$ and 0.84 (LR). Due to high fuel consumption during this phase and thus a decrease of the overall aircraft weight, a step-climb or continuous cruise-climb can be chosen.

The Descent phase begins at Top of Descent (TOD) altitude after the cruise. The air speeds during descent can be defined by a given air speed profile. The speed (Ma) remains constant until the crossover altitude is reached, which is fixed at $30000 \mathrm{ft}$. After falling below the crossover altitude, the speed is kept constant at $300 \mathrm{kt}$ (CAS). Similar to the climb phase, the speed has to be reduced to $250 \mathrm{kt}$ (CAS), while reaching $10000 \mathrm{ft}$. The following rates of descents can be defined according to Allen ${ }^{13}$. For simplification, it is assumed that the approach angle of $3^{\circ}$ is constant during descent.

The start of the Approach is defined by the Initial Approach Fix (IAF) point, which marks the beginning of the approach at the altitude of $4000 \mathrm{ft}$. After reaching the Final Approach Fix (FAF), the final approach begins. Deceleration phases during the approach are defined at the IAF and FAF. The Landing phase begins with the threshold crossing at $100 \mathrm{ft}$ and is followed by the touchdown at defined touchdown speeds. The following Taxi-in with a typical speed between 15-20 kt lasts about 5 minutes to reach the final parking position.

\section{Aircraft System Models}

For estimating the secondary power demand of aircraft systems within early aircraft design phases, two different approaches are applied. On the one hand, simplified physical models with defined input parameters and system constraints are developed. On the other hand, semi-empirical approaches or data found in published literature are used for power requirement estimations. Figure 3 gives an overview on the considered aircraft systems and defines the system boundary on aircraft level within this research study. The transport aircraft propulsion (power) system can be divided into primary power for generating thrust and into secondary power to supply the onboard distributed aircraft systems. In general, the power generation and distribution system provides three different power system architectures (networks): electrical power, hydraulic power and pneumatic power.

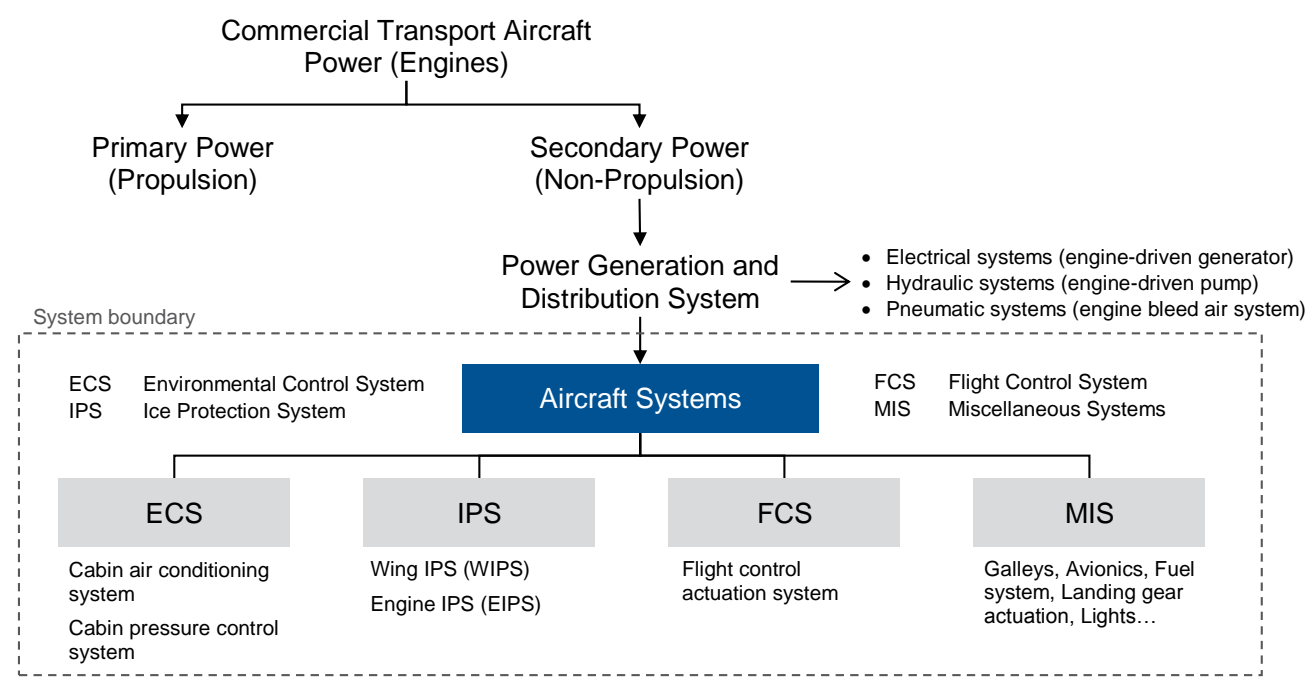

Figure 3. Overview on considered aircraft systems for secondary power requirement estimations.

In this contribution, simplified system models are used for the three most important aircraft systems, regarding power consumption and criticality: The Environmental Control System (ECS), the Ice Protection System (IPS) and the Flight Control System (FCS). On basis of aircraft-level functions, major functions on system level are derived and used for building up system models. The power requirements of the remaining, miscellaneous systems (MIS), e.g. galleys, avionics, and fuel systems, are mainly based on empirical methods or existing data from literature 


\section{A. Environmental Control System}

The main functions of the ECS of a transport aircraft are cabin air conditioning and cabin pressurization, in order to provide a cabin environment that is comfortable for the crew and passengers. Furthermore, avionics and electrical airframe systems have to be cooled as well. To meet the requirements in early transport aircraft design phases, a simplified pneumatic and electrical ECS model is developed.

Figure 4 shows the Air Conditioning System (ACS) model with corresponding mass flow rates and heat flow rates. The pneumatic ECS is supplied by engine bleed air system (including the pre-cooler). The bleed air is generally drawn off from the intermediate pressure compressor or the high pressure compressor of the engine, regulated down by valves and pre-coolers ${ }^{14}$. Therefore, the temperature $T_{\text {bleed }}$ of the bleed air supply is assumed to be constant throughout the mission - regardless of the engine speed. Whereas for MEA with no-bleed architectures, ram air is used, passing electrically-driven Cabin Air Compressors (CAC). The resulting compressed air requires no down regulation before entering the Air Conditioning Pack (ACP) ${ }^{3}$. For the bleed-less architecture the temperature $T_{\text {ram }}$ of the ram air supply changes with flight altitude and speed of the aircraft.

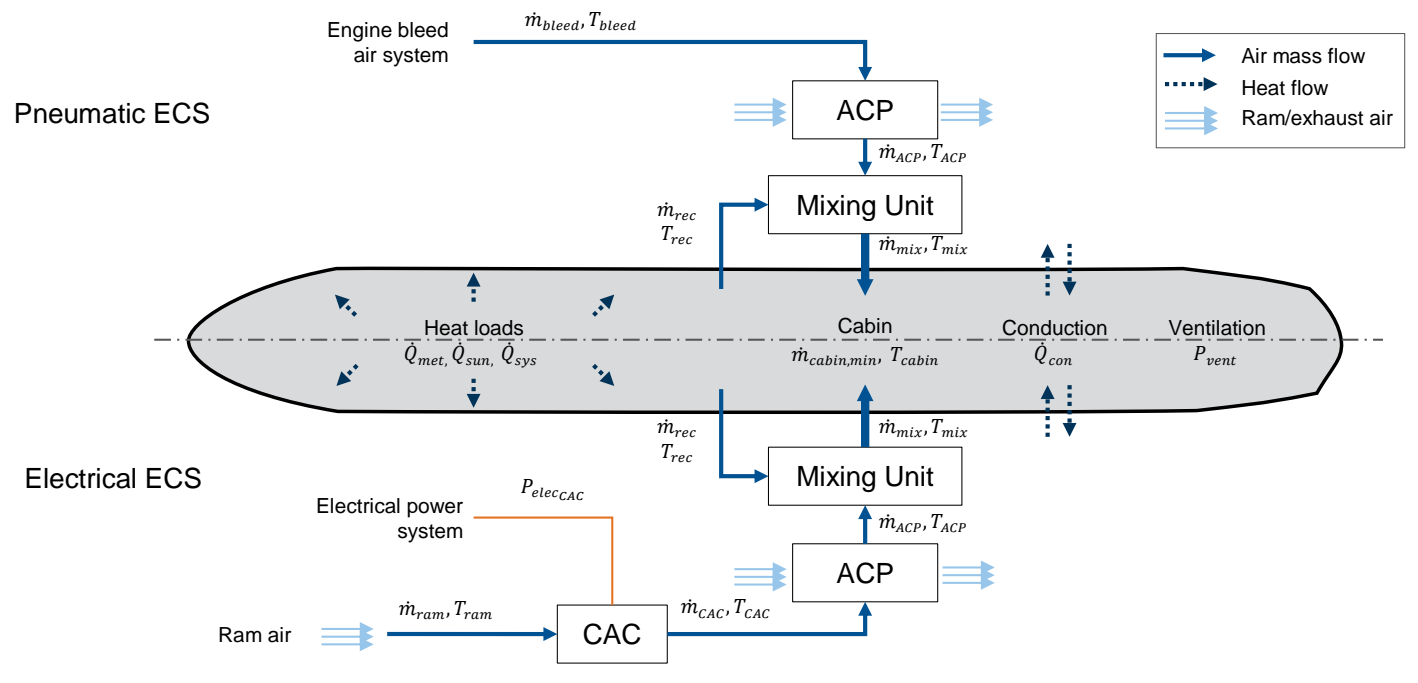

Figure 4. Simplified Air Conditioning System (ACS) model for a pneumatic (top) and an electrical (bottom) Environmental Control System (ECS)

The CAC is electrically driven by an adjustable speed motor, requiring electrical power $P_{\text {elec }}{ }_{C A C}$. As for the bleed air supply, the temperature $T_{C A C}$ after the CAC is also assumed as constant. According to Moir and Seabridge ${ }^{15}$, typical temperature values are $T_{\text {bleed }} \approx 200{ }^{\circ} \mathrm{C}$ and $T_{C A C} \approx 95^{\circ} \mathrm{C}$ before entering the ACP. The ACP consists of an air cycle machine and a heat exchanger using ram air, to change the hot pressurized air to cold air for air conditioning ${ }^{14}$. Nevertheless, for this simplified ECS model both, the CAC and the ACP are assumed as a black box with characteristic efficiency factors $\eta_{C A C}$ and $\eta_{A C P}$.

Within the mixing unit - assumed as loss-free - recirculated cabin air is mixed with the conditioned supply air from the ACP and directed to the cabin by overhead distribution nozzles. The recirculated mass flow rate $\dot{m}_{\text {rec }}$ provides about $40-50 \%{ }^{5,16}$ of the cabin air flow rate $\dot{m}_{\text {cabin }}$. The recirculation factor $f_{\text {rec }}$ is initially set 0.5 , but can vary during the flight mission. Furthermore, it is assumed that the temperatures $T_{A C P}, T_{r e c}$, and $T_{m i x}$ are equal to the required cabin air temperature $T_{\text {cabin }}$. It is assumed that the cabin temperature is constant and homogenous inside the cabin. A hot air manifold with trim air (low pressure, high temperature) to adjust the temperature in different cabin zones is not considered.

The required power for the ACS is defined as the sum of all heat flow rates, see Eq. 3. The total heat flow rate results from different heat loads and from the heat transfer between the cabin and the environment $\dot{Q}_{\text {con }}$ (conduction). The heat loads can be divided in heat dissipation by passengers and crew $\dot{Q}_{m e t}$ (metabolism), solar heating by the sun $\dot{Q}_{s u n}$ and the heat loads by several electrical systems $\dot{Q}_{s y s}$, see Table 4 . in the appendix.

$$
P_{A C S} \stackrel{\text { def }}{=} \sum \frac{d Q_{i}}{d t}=\dot{Q}_{c a b i n}=\dot{Q}_{c o n}+\dot{Q}_{m e t}+\dot{Q}_{s y s}+\dot{Q}_{s u n}
$$


The supply requirements for air conditioning (index with $A C S$ ) of the pneumatic and electrical ECS can be calculated by using Eq. 4, where $\eta_{A C P}$ represents the efficiency of the ACP and $c_{p}$ the specific heat capacity of air.

$$
\dot{m}_{\text {bleed } / \mathrm{ram}_{A C S}}=\dot{m}_{A C P_{A C S}}=\frac{P_{A C S}}{\eta_{A C P} \cdot c_{p} \cdot\left(T_{\text {bleed } / \mathrm{ram}}-T_{A C P}\right)}
$$

Besides air conditioning requirements, the fresh air requirement for crew and passengers must be fulfilled. The FAA (FAR 25.831) specifies that the minimum fresh air $\dot{m}_{\text {fresh }}$ provided by the ventilation system must be at least $0.55 \mathrm{lbs} / \mathrm{min}$ per occupant. According to Lammering ${ }^{5}$, further values of $0.75 \mathrm{lbs} / \mathrm{min}$ (standard) and $1.0 \mathrm{lbs} / \mathrm{min}$ (comfort) are common. Following this, the mixed air mass flow rate $\dot{m}_{\text {mix }}$ can be calculated to verify if the fresh air requirements are met. If the mass flow rate for air conditioning is too low $\left(\dot{m}_{m i x_{A C S}}<\dot{m}_{f r e s h}\right)$, corrected supply mass flow rates are calculated, see Eqs. 5 and 6.

$$
\begin{aligned}
& \dot{m}_{m i x_{A C S}}=\dot{m}_{A C P_{A C S}}+f_{\text {rec }} \cdot \dot{m}_{\text {fresh }} \\
& \dot{m}_{\text {bleed } / \text { ram }}=\dot{m}_{A C P}= \begin{cases}\dot{m}_{\text {bleed } / \text { ram }_{A C S}}+\left(\dot{m}_{\text {fresh }}-\dot{m}_{\text {mix }} \text { ACS }\right), & \dot{m}_{m i x_{A C S}}<\dot{m}_{\text {fresh }} \\
\dot{m}_{\text {bleed } / \text { ram }_{A C S}}, & \dot{m}_{\text {mix }} \geq \dot{m}_{\text {fresh }}\end{cases}
\end{aligned}
$$

The resulting amount of bleed air $\dot{m}_{\text {bleed }}$ represents the power requirements for the pneumatic ECS. The electrical power requirements for the CAC are calculated using Eq. 7. Additionally, for both the pneumatic and electrical ECS, the power for ventilation $P_{\text {vent }}$ must be considered. Here, the ventilation power is estimated with $135 \mathrm{~W}$ per occupant ${ }^{17}$.

$$
P_{\text {elec } C A C}=\frac{\dot{m}_{C A C} \cdot c_{p} \cdot\left(T_{C A C}-T_{\text {ram }}\right)}{\eta_{C A C}} \quad \text { with } \dot{m}_{C A C}=\dot{m}_{\text {ram }}
$$

As mentioned above, the ECS provides cabin pressurization as well. The cabin pressurization is achieved by controlling the outflow of the cabin via controlled outflow valves. In the isobaric mode, the cabin altitude (pressure) is controlled to a predetermined value. When the maximum differential pressure is reached, the mode is automatically switched to the constant differential mode. In this mode, the difference between cabin pressure and ambient air pressure is kept constant. In this study, the power requirements for the electrical controlled outflow valves is neglected, as it is considerably less than the power requirements for air conditioning. Nevertheless, for the sake of completeness, the ECS system model calculates the cabin altitudes, considering both modes of pressurization.

\section{B. Ice Protection System}

The Ice Protection System (IPS) has the main function to avoid ice formation or ice accumulations at aerodynamically sensitive areas such as wing leading edges and engine inlets (cowling $)^{15}$. Furthermore, air data probes, cockpit windows, antennas and valves are heated to prevent icing and thus malfunctions or failures. In general, hot air from the engine bleed air system or electrical heating elements are used for anti-icing or de-icing ${ }^{14}$.

The two main IPS power consumers are the Engine IPS (EIPS) and Wing IPS (WIPS). EIPS is generally supplied by bleed air, even for MEA architectures like the B787. For the WIPS two possible architectures are considered: The pneumatic supplied and the electrical supplied WIPS. The engine bleed air system supplies the pneumatic WIPS, where the leading-edge devices (e.g. slats) are heated over piccolo tubes with the hot bleed air, see Fig. 5 on the left. The electro-thermal WIPS is supplied by the electrical power system. Therefore, attached heating mats inside the leading-edge devices provide the required heating for anti-icing or de-icing, see Fig. 5 on the right.

Sherif et al. ${ }^{18}$ presented a semi-empirical method for local heat transfer and ice accretion on aircraft wings, where the local heat transfer is influenced by energy added or removed to the surface in form of different heat flux densities. Based on this method and according to Lammering ${ }^{5}$ and Chakraborty ${ }^{16}$, the total heat flux $\dot{q}_{\text {total }}$ is simplified and can be calculated using Eq. 8 .

$$
\dot{q}_{\text {total }}=\dot{q}_{c o n v}+\dot{q}_{\text {sens }}+\dot{q}_{\text {evap }}-\dot{q}_{\text {kin }}
$$

The convectional heat flux $\dot{q}_{\text {conv }}$ describes the cooling of the surface due to air flow. The sensible heating $\dot{q}_{s e n s}$ is the heat flux required to heat the impinging water droplets to the desired surface temperature. Evaporation at wing or engine cowling and the corresponding heat losses are considered over the heat flux $\dot{q}_{\text {evap }}$. Furthermore, $\dot{q}_{\text {kin }}$ represents the kinetic heating. The corresponding calculations are shown in Table 5 in the appendix. 

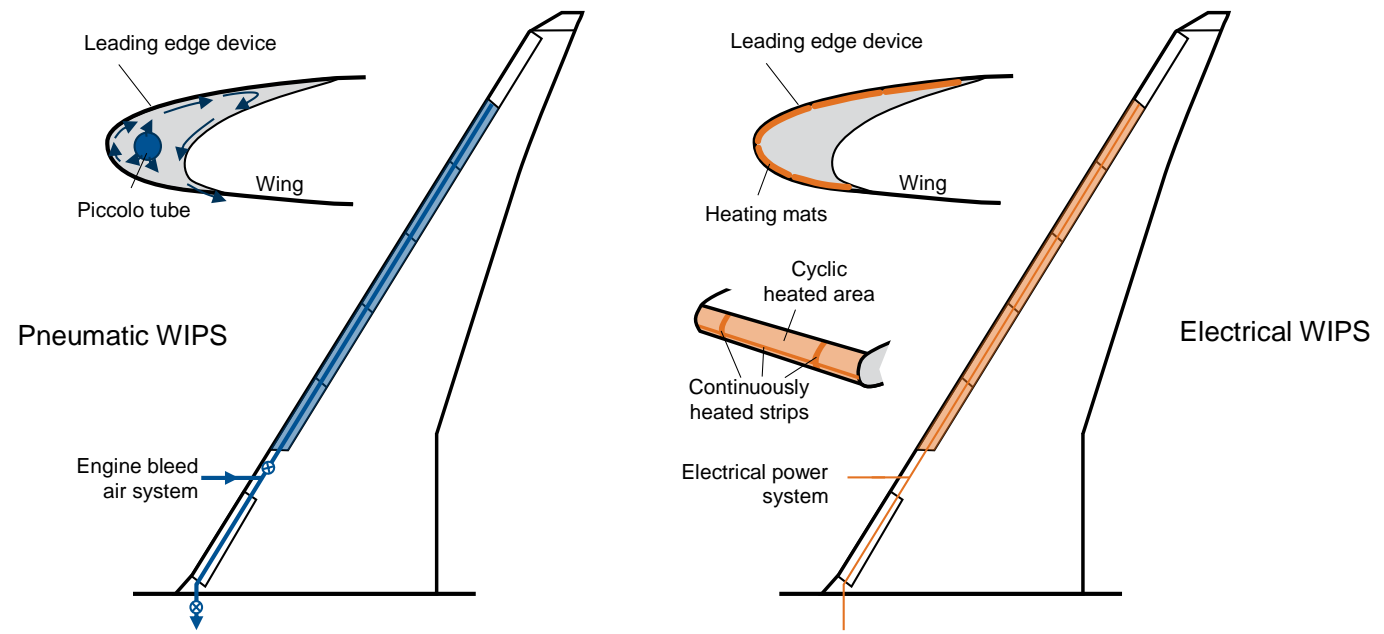

\section{Figure 5. Simplified Wing Ice Protection System (WIPS) models with pneumatic supply (left) and electrical power supply (right) (adapted from Chakraborty ${ }^{19}$ and Lammering ${ }^{5}$ ).}

In general, the required bleed air mass flow for a pneumatic IPS is calculated using Eq. $9^{16}$, considering the surface area to protect $A_{\text {prot }}$ and the desired temperature of the protected surface $T_{\text {surface }}$. $T_{\text {bleed }}$ is the temperature of the bleed air supply and $\eta_{\text {bleed }}$ IPS is the overall heat transfer efficiency for the pneumatic IPS, that is assumed to be $65 \%{ }^{5}$.

$$
\dot{m}_{\text {bleed }}=\frac{\dot{q}_{\text {total }} \cdot A_{\text {prot }}}{\eta_{\text {bleed }_{I P S}} \cdot c_{p} \cdot\left(T_{\text {bleed }}-T_{\text {surface }}\right)}
$$

To calculate the power for the electrical IPS two further steps are required. Firstly, the resulting heat flux $\dot{q}_{\text {total }}$ elec of the electrical WIPS can be calculated using Eq. 10. The parting strips are continuously heated (see Fig. 5) for anti-

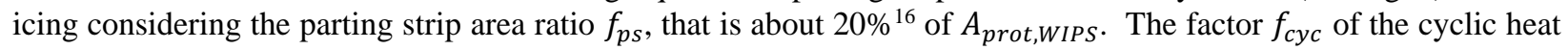
flux term is assumed to be $0.05^{20}$, which describes the ratio of heating time to the cycling time. The heat flux $\dot{q}_{c y c}$ for de-icing of the cyclic heated area is estimated with Eq. $11^{16}$.

$$
\begin{gathered}
\dot{q}_{\text {total }_{\text {elec }}}=\dot{q}_{\text {total }} \cdot f_{p s}+\dot{q}_{c y c} \cdot f_{c y c} \\
\dot{q}_{c y c}=\frac{\rho_{\text {ice }} \cdot l_{\text {ice }}}{t_{\text {heat }}} \cdot\left(c_{\text {ice }} \cdot\left(T_{\text {ref }}-T_{\infty}\right)+L_{f}\right)
\end{gathered}
$$

Therefore, it is assumed that a thin layer of ice $l_{i c e}$ of $0.5 \mathrm{~mm}$ is intermittently melted and heated to the desired surface temperature. $L_{f}$ is the latent heat that arises during evaporation of water.

Secondly, the power requirements $P_{\text {WIPS }}$ for the electrical WIPS can be calculated, see Eq. 12. A typical values of $\eta_{\text {elec }_{I P S} \text { is }} 70-75 \%{ }^{16}$. As mentioned above, additional electrical power $P_{\text {others }}$ estimations for heating of other devices (e.g. air data probes, cockpit window) are required.

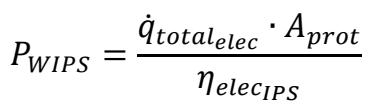

According to the Jeck ${ }^{21}$ (FAA) different icing design envelopes ${ }^{*}$ are considered for the wing ice protection and for engine ice protection: Continuous maximum atmospheric icing conditions for WIPS and intermittent maximum conditions for EIPS. Icing design envelopes are characterized by ambient temperature vs. pressure altitude. If the aircraft

\footnotetext{
${ }^{*}$ Icing design envelopes are characterized by ambient temperature vs. pressure altitude (Jeck ${ }^{21}$ pp. 2-4)
} 
is within the icing conditions the WIPS or EIPS are activated, respectively. For both, a standard median volume diameter of the water droplets of $20 \mu \mathrm{m}$ is assumed ${ }^{21}$, but can be varied as input parameter within the implemented tool.

The estimated electrical power for the heating of other system (e.g. cockpit window heating, air data probes) are based on values published by Roskam ${ }^{22}: 6 \mathrm{~kW}$ during take-off and climb, $7.5 \mathrm{~kW}$ during cruise and again $6 \mathrm{~kW}$ during descent and landing.

\section{Flight Control System}

The Flight Control System (FCS) of commercial transport aircraft can be divided into a configurational system and architectural system ${ }^{6}$. The FCS configuration includes the type, allocation and positions of flight control devices. The FCS architecture defines the actuation of the devices, the redundant distribution of the power supply and the assignment of the flight control computers for reconfiguration.

For this study, conventional and simplified FCS configurations for both baseline aircraft (MR/LR) are defined, see Fig. 6. Based on the defined FCS configurations, the FCS architecture can be derived. For reasons of redundancy each aileron and each elevator is actuated by two actuators. The actuation system of the rudder at the vertical tail plane consists of three actuators. The wing leading and trailing edge devices for high-lift are actuated over transmission shafts, which are driven by central Power Control Units (PCU).

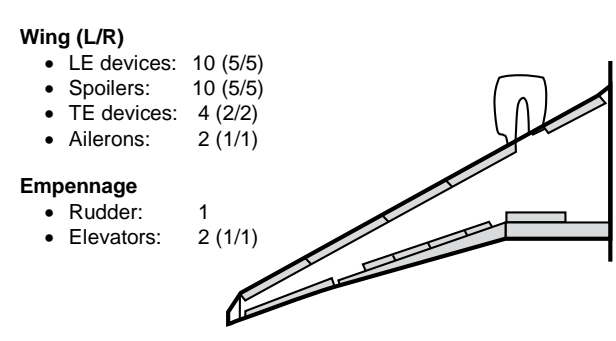

Medium-Range (MR)

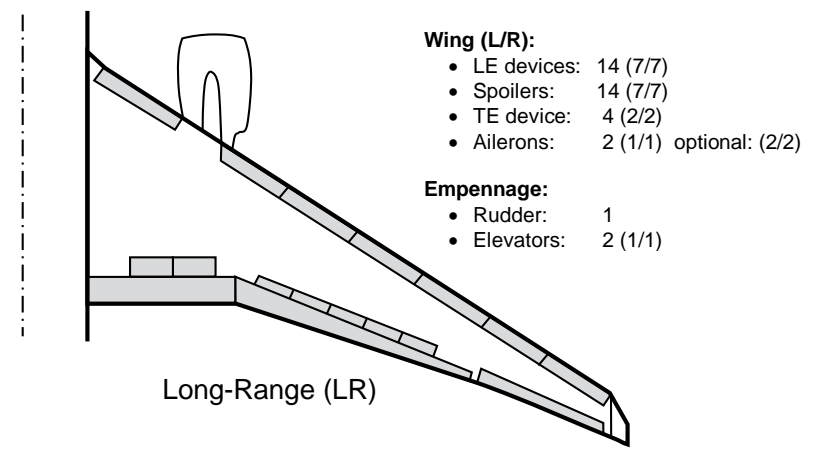

Long-Range (LR)

Figure 6. Schematic of the FCS configurations of the baseline aircraft (MR/LR).

According to Lammering ${ }^{5}$, the power requirements of the FCS strongly depends on the aerodynamic forces acting on the devices. Therefore, the power estimations can be done independent from the chosen actuation technology. The power requirements $P_{C S}$ for the actuation of flight control surfaces - including ailerons, rudder and elevators - are estimated over the surface loads in form of hinge moments $M_{\text {hinge }}$, which are required for the desired deflection rates $\dot{\gamma}_{C S}$, see Eq. $13^{5}$.

$$
P_{C S}=M_{\text {hinge }} \cdot \dot{\gamma}_{C S}
$$

The hinge moment is proportional to the stagnation pressure $\frac{\rho_{\infty}}{2} \cdot V_{T A S}^{2}$, the surface area $A_{C S}$, the average surface chord $\bar{c}_{C S}$, and the hinge moment coefficient $C_{\text {hinge,CS}}$, see Eqs. 14 and $15^{23}$. To consider compressible effects at speeds, the coefficients $C_{\text {hinge, } \alpha}, C_{\text {hinge, } \gamma}$ are divided by the Prandtl-Glauert factor $\beta=\sqrt{1-M a^{2}}$. The angle of attack $\alpha$ and the deflection angle $\gamma$ are dependent on the mission flight phases.

$$
\begin{gathered}
M_{\text {hinge }}=\frac{\rho_{\infty}}{2} \cdot V_{T A S}^{2} \cdot A_{C S} \cdot \bar{c}_{C S} \cdot C_{\text {hinge }, C S} \\
C_{\text {hinge }, C S}=C_{\text {hinge }, 0}+C_{\text {hinge }, \alpha} \cdot \alpha+C_{\text {hinge }, \gamma} \cdot \gamma
\end{gathered}
$$

The deflection rates $\dot{\gamma}_{C S}$ of the control surfaces are calculated by normalized maximum deflection angles $\hat{\delta}_{+} / \hat{\delta}_{-}$ and the related CPS (Cycles per Second) for each phase of the mission, see Table 2. One CPS describes the deflection of the control surface and its return to zero position within one second. 
The resulting power requirements result from the actuation efficiency, see Eq. 16. For this simplified FCS model, architectures with Servo-valve controlled Hydraulic Actuator (SHA) and the Electro-Hydrostatic Actuator (EHA) are considered.

$$
P_{\text {hyd/elec } C S}=\frac{P_{C S}}{\eta_{S H A / E H A}}
$$

Table 2. Characteristics of control surface deflections and related Cycles Per Second (CPS) for each mission phase (based on Simsic ${ }^{8}$ and Chakraborty ${ }^{16}$ ).

\begin{tabular}{|c|c|c|c|c|c|}
\hline Mission Phase & $C P S$ & $\begin{array}{c}\text { Aileron } \\
\hat{\delta}_{+} / \hat{\delta}_{-}\end{array}$ & $\begin{array}{c}\text { Elevator } \\
\hat{\delta}_{+} / \hat{\delta}_{-}\end{array}$ & $\begin{array}{c}\text { Rudder } \\
\hat{\delta}_{+} / \hat{\delta}_{-}\end{array}$ & $\begin{array}{c}\text { Spoiler } \\
\hat{\delta}\end{array}$ \\
\hline Taxi-out $^{a}$ & 0.00 & $0.00 /-0.00$ & $0.00 /-0.00$ & $0.00 /-0.00$ & 0.00 \\
\hline Take-off & 0.40 & $0.12 /-0.12$ & $0.20 /-0.20$ & $0.20 /-0.20$ & 0.12 \\
\hline Initial climb & 0.40 & $0.12 /-0.12$ & $0.20 /-0.20$ & $0.20 /-0.20$ & 0.12 \\
\hline Climb 1-3 & 0.40 & $0.12 /-0.12$ & $0.20 /-0.20$ & $0.20 /-0.20$ & 0.12 \\
\hline Cruise $^{b}$ & 0.20 & $0.12 /-0.12$ & $0.20 /-0.20$ & $0.20 /-0.20$ & 0.12 \\
\hline Descent 1-3 & 0.30 & $0.12 /-0.12$ & $0.20 /-0.20$ & $0.20 /-0.20$ & 0.12 \\
\hline Initial Approach & 0.30 & $0.12 /-0.12$ & $0.20 /-0.20$ & $0.20 /-0.20$ & 0.12 \\
\hline Final Approach & 0.40 & $0.32 /-0.40$ & $0.50 /-0.50$ & $0.50 /-0.50$ & 0.30 \\
\hline Landing & 0.40 & $0.32 /-0.40$ & $0.50 /-0.50$ & $0.50 /-0.50$ & 0.40 \\
\hline Taxi-in & 0.00 & $0.00 /-0.00$ & $0.00 /-0.00$ & $0.00 /-0.00$ & 0.00 \\
\hline
\end{tabular}

The hinge moments of high-lift devices cannot be determined only with geometry data of the leading and trailing edge devices, but also require knowledge of the kinematics of the associated mechanism. Thus in this contribution, the power requirements for the high-lift system actuation is based on an empirical approach, according to Chakraborty ${ }^{16}$.

In this work, the author relates the installed PCU power to the maximum take-off mass MTOM of existing commercial transport aircraft to establish following Eq. 17.

$$
\begin{gathered}
P_{P C U}=1196.9 \cdot\left(\frac{M T O M}{1000}\right)^{0.5551} \\
P_{h y d_{P C U}}=\frac{P_{P C U}}{\eta_{P C U}}
\end{gathered}
$$

The result represents the total power for both PCUs for the leading edge and trailing edge devices, and shows good accordance to power data presented in the work of Lammering ${ }^{5}$. Finally, the required hydraulic power for the PCU can be calculated using Eq. 18, where $\eta_{P C U}$ is the PCU efficiency.

\section{Miscellaneous Systems}

All remaining system with considerable power requirements which cannot be assigned to the ECS, IPS or FCS, are defined under miscellaneous systems (MIS), see Table 6 in the appendix. The hydraulic power requirements for retraction and deployment of the landing gear is estimated by Eq. 19, using the hydraulic power of the PCU as a reference.

$$
P_{h y d_{L G}}=1.53 \cdot P_{h y d_{P C U}}
$$

This estimation is based on the hydraulic power requirements from flight test data of an Airbus A320 presented in the work of Lammering 5 . For simplification, it is assumed that the power demand for retraction and extension is equal.

\section{Simulation Framework}

The presented parameterized mission and the aircraft system models for secondary power requirement estimations are implemented into a MATLAB ${ }^{\circledR}$ tool. Within this framework, mission objects and system objects for the FCS, ECS, IPS, and MIS are initialized, see Fig 7. The mission object, consisting of phases and steps, is automatically generated and initialized based on mission input parameters, set ISA conditions and altitude step size. The aircraft system objects are strongly based on the system models from section III. This object-oriented approach allows the easy addition of 
flight phases and the implementation of new system architectures, technologies or concepts. After the mission and system objects are initialized, the system activities, including icing conditions (IPS) and duty cycles of flight control surfaces (FCS), are checked. Finally, the secondary power requirements are calculated for all steps of each phase.

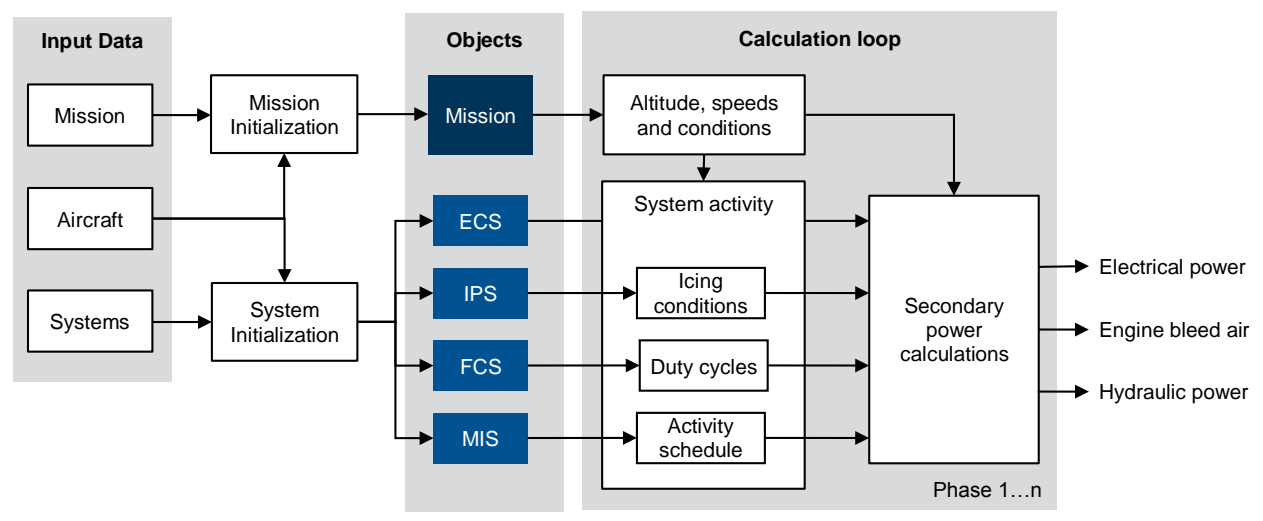

Figure 7. Top-level schematic of the simulation framework with implemented mission and system objects.

\section{Case Study and Results}

The case study considers two configuration of the Medium-Range (MR) baseline aircraft with 162 passengers on board and a mission flight range of $3000 \mathrm{~nm}$. The cruise speed is constant at Mach 0.81 . The first configuration has a conventional bleed-air architecture on board to supply the ECS and IPS. Whereas, the second configuration is a MEA with electrically supplied ECS and IPS. In the following sections, the individual results of the secondary power requirements of the considered aircraft systems are viewed in detail. Therefore, the baseline aircraft and the altitude and speed profile results of the reference mission serve as input data.

\section{A. Reference Mission}

The flight mission is performed at standard ISA conditions, starting and landing at Mean Sea Level (MSL). The altitude step size is set to $500 \mathrm{ft}$. Figure 9 shows the altitude and speed profile of the overall mission. The overall mission time calculate is about $418 \mathrm{~min}$. The cruise segment is performed as a step-climb, starting at $36000 \mathrm{ft}$ (TOC) and ending at $40000 \mathrm{ft}$ (TOD).
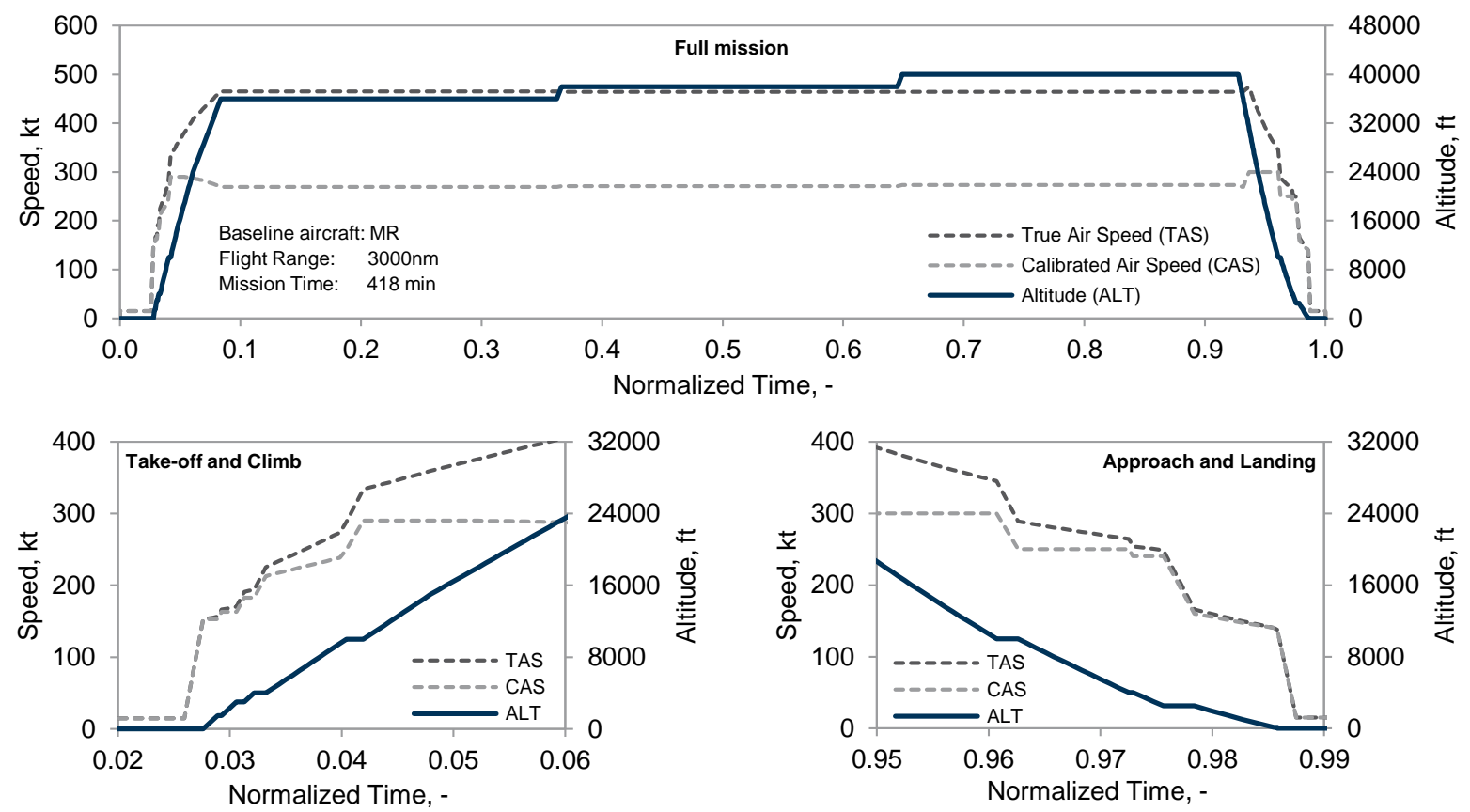

Figure 9. Flight mission results for the medium-range baseline aircraft as a function of time.

10

American Institute of Aeronautics and Astronautics 


\section{B. Environmental Control System}

The ECS is among the largest consumers of secondary power. For the following investigation, a constant cabin temperature of $24{ }^{\circ} \mathrm{C}$ and a constant fresh air demand for the cabin of $0.75 \mathrm{lb} / \mathrm{min}$ per occupant (passengers and crew) is set. Figure 7 shows the results of the cabin heat flows (left) and the secondary power requirements for the pneumatic and electrical ECS (right). For most of the flight mission time during cruise, the resulting cabin heat flows hardly vary and accordingly the power requirements in form of engine bleed air and electrical power remain constant. Furthermore, two effects can be recognized: Firstly, during flight phases of rapid altitude changes (climb/descent) and thus significant ambient temperature changes, noticeable increase/decrease of the conductive heat flow can be recognized. Secondly, after cruise, a rapid reduction in system heat flow can be seen after the cruise phase, due to the deactivation of the galleys.
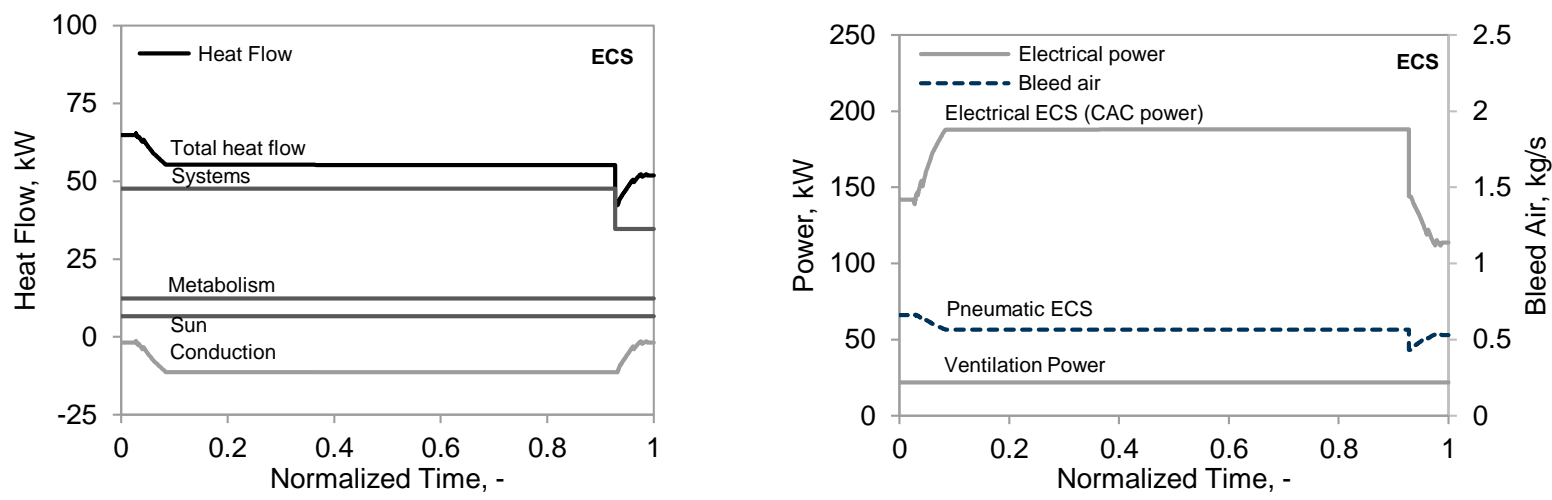

Figure 8. Cabin heat flows and secondary power requirements of the pneumatic and electrical Environmental Control System (ECS).

The breakdown of averaged power requirements of each mission phase of the ECS are shown in Fig. 9. The maximum bleed air demand for the pneumatic ECS is required for air conditioning on ground. Whereas, for the electrical ECS the power requirements increase with higher altitudes and a maximum can be observed during cruise. This can be explained by the decreasing air temperatures and thus decreasing temperatures of the ram air, supplying the CAC, see also Eq. 7.
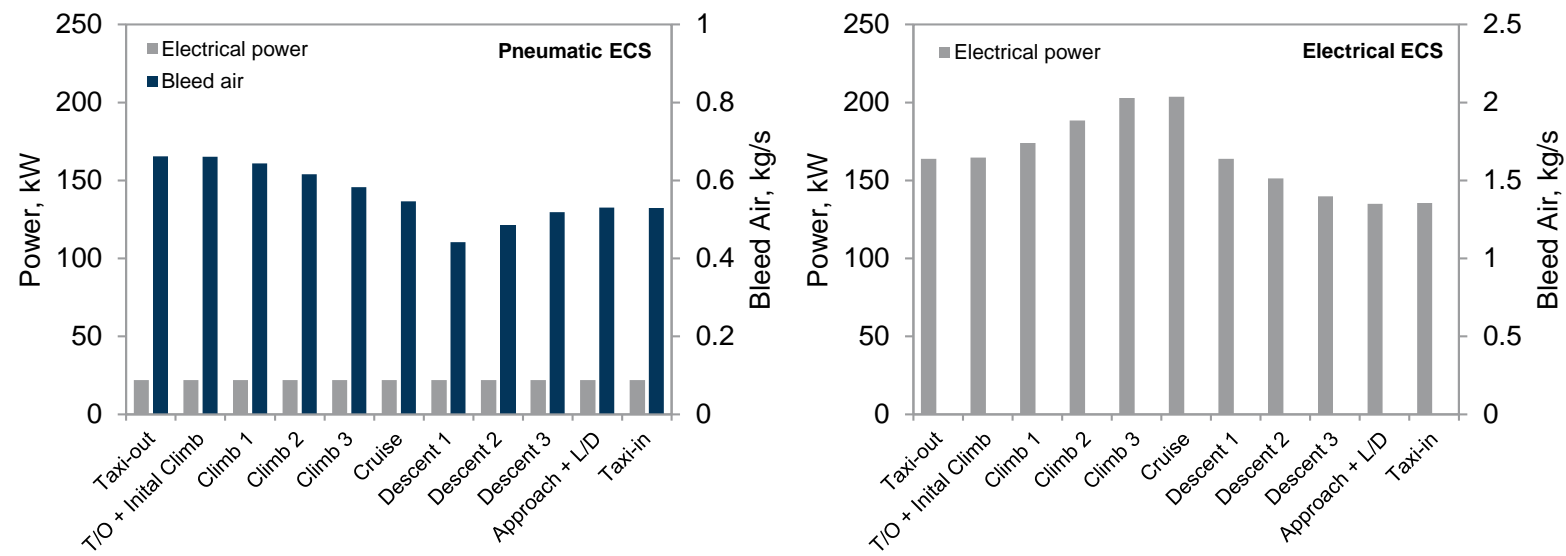

Figure 9. Averaged power requirements of the pneumatic (left) and electrical (right) Environmental Control System (ECS).

\section{Ice Protection System}

The IPS is also one of the largest secondary power consumers on board of the aircraft. The desired surface temperatures are set to $6{ }^{\circ} \mathrm{C}$ for the WIPS (de-icing) and $36{ }^{\circ} \mathrm{C}$ for the EIPS (anti-icing). The protecting areas of the MR baseline aircraft are $7.5 \mathrm{~m}^{2}$ for the wing leading edge devices and $5.4 \mathrm{~m}^{2}$ for both engine cowlings.

The results of the pneumatic and electrical IPS power requirements mission are presented in Fig. 10 on the top. It can be observed two "peaks" of engine bleed air demand during climb and descent phases occur. With increasing altitude and decreasing temperature during climb, the aircraft enters the icing condition envelope of the EIPS and 
WIPS, see Fig. 10 on the bottom. Similar behavior is observed for the descent phase. During cruise the aircraft leaves the icing envelopes again, thus no IPS is activated. The higher bleed air demand for the EIPS results on the one hand from the higher surface temperature of $36{ }^{\circ} \mathrm{C}$ and on the other hand on the higher liquid water content for intermittent maximum conditions considered, see also section III/B. The bleed air demand for the EIPS remains the same, since the EIPS is still supplied by engine bleed air. As expected, the now electrically supplied WIPS shows the same behavior as the pneumatic WIPS, see Fig. 10 at the bottom.
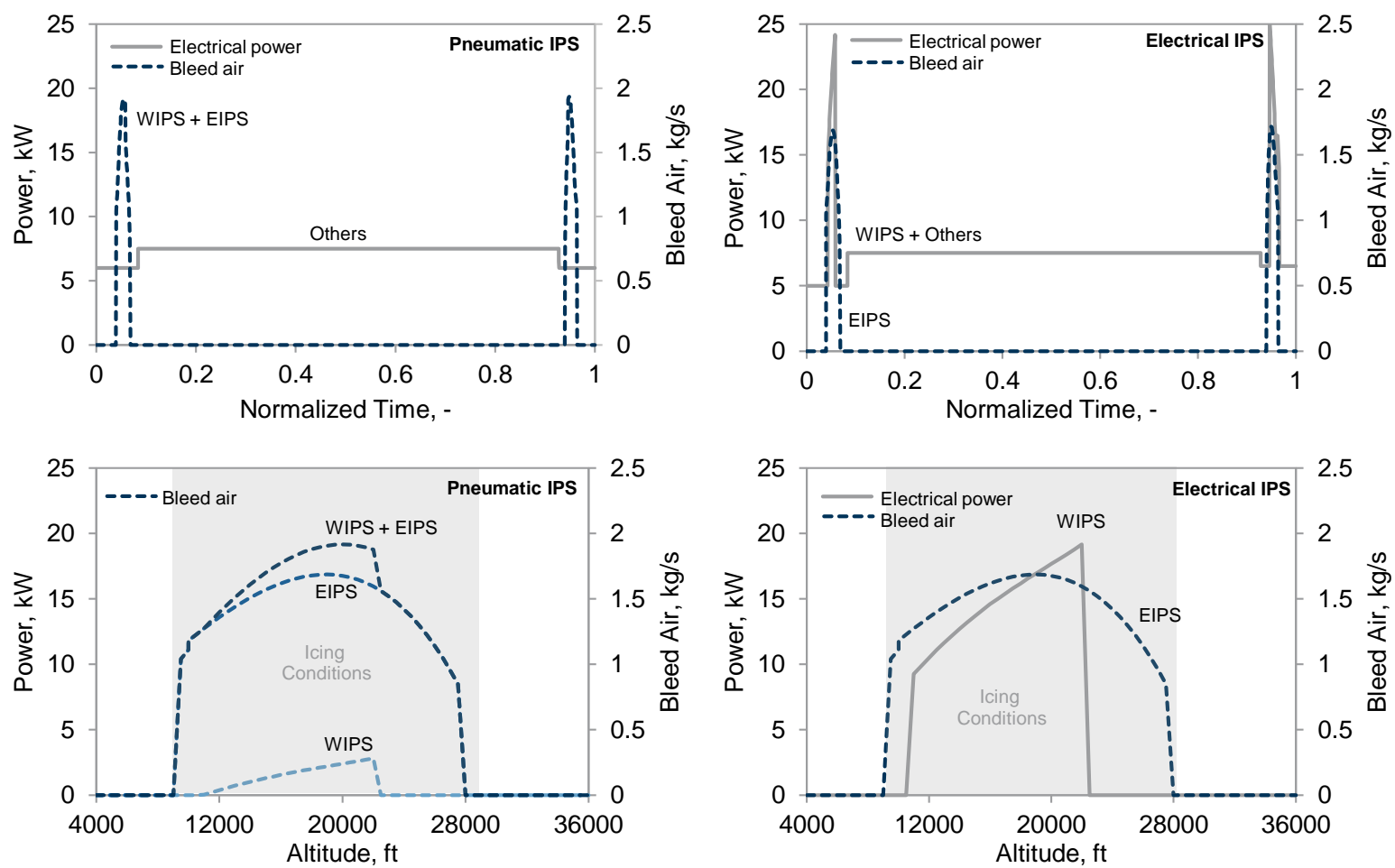

Figure 10. Power requirements of the pneumatic (left) and the electrical (right) Ice Protection System (IPS).

The averaged secondary power requirements for each flight phase are displayed in Fig. 11. Both, the pneumatic and electrical IPS show secondary power requirements during climb and descent, where icing conditions occur. The results for the WIPS and EIPS show good accordance to the results for a B737-800 type of aircraft ${ }^{23}$ and to the results of an $\mathrm{A} 320^{5}$.
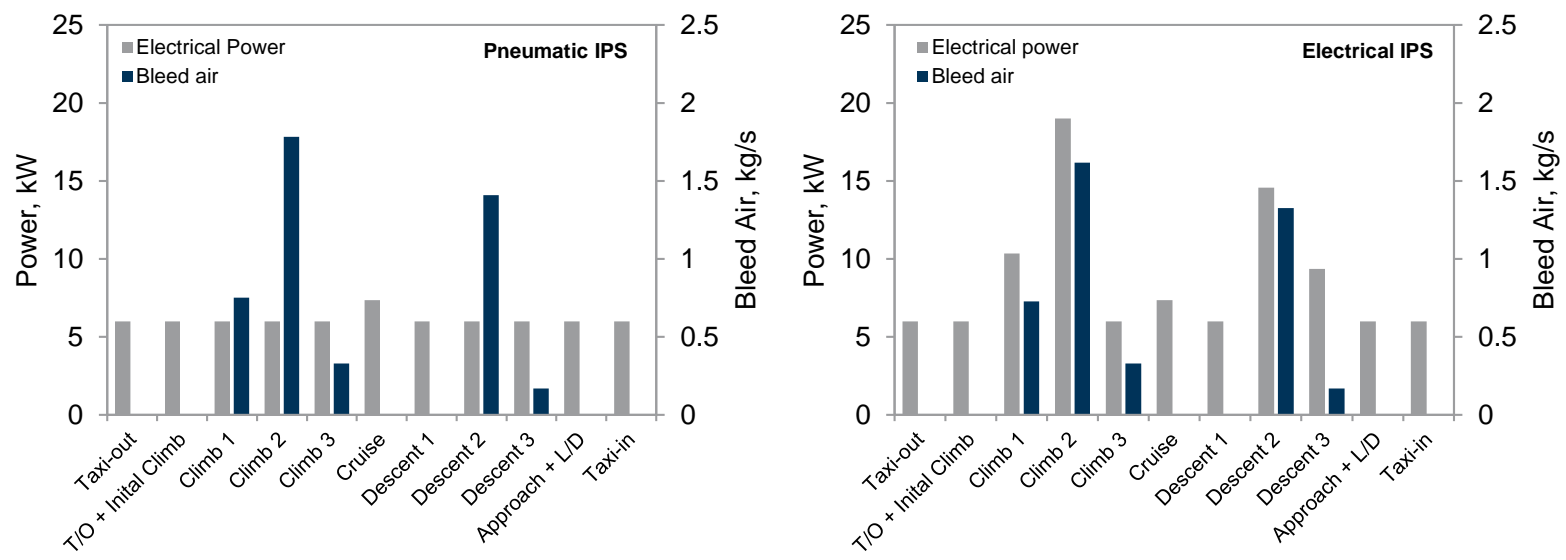

Figure 11. Averaged power requirements of the pneumatic (left) and electrical (right) Ice Protection System (IPS). 


\section{Flight Control System Power}

For this case study, two different FCS actuation systems are defined for the ailerons, rudder, elevators and spoilers. For the conventional baseline aircraft, the actuation system consists of well-known Servo-valve controlled Hydraulic Actuators (SHA), with an assumed efficiency of $\eta_{S H A}=60 \%$. In contrast, Electro-Hydrostatic Actuators (EHA) are used for the MEA baseline aircraft $\left(\eta_{E H A}=85 \%\right)$, similar to the actuation architecture presented by Chakraborty ${ }^{23}$. Nevertheless, the leading-edge and trailing-edge devices of the high-lift system are actuated by centrally hydraulicpowered PCUs.

In Fig. 12 on the left, the required mean actuation power of the flight control devices over the full mission are depicted. It can be seen, that for the descent, approach and landing phases, the actuation power increases. This is due to the higher cycles per second (CPS) of each control surface and due to higher required deflections or deflection rates, respectively. The short term hydraulic power required for the PCUs of the high-lift system (not shown in the graphs) is approximately $22 \mathrm{~kW}$. The averaged secondary power requirements for FCS with hydraulic/electrical (SHA/EHA) architectures for each phase are displayed in Fig. 12 on the right.
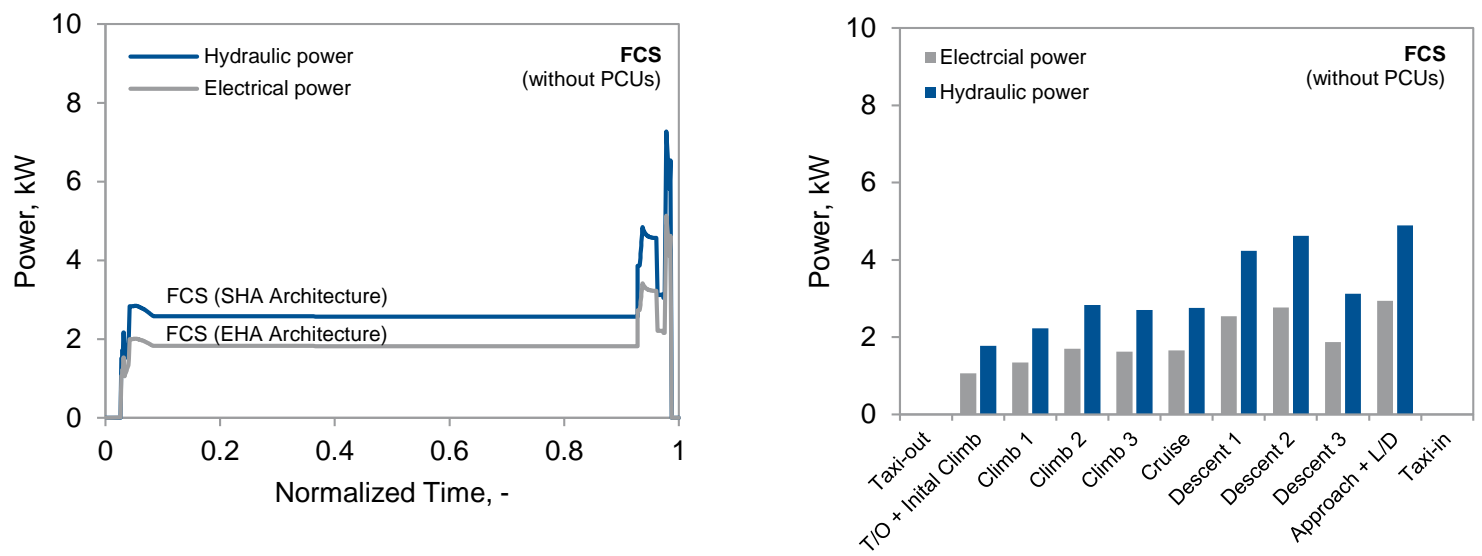

Figure 12. Mean actuation power requirements (left) and averaged secondary power requirements for each phase (right) of the Flight Control System (FCS).

\section{E. Miscellaneous Systems Power}

The remaining systems, relevant for secondary power consumption, are defined as Miscellaneous Systems (MIS). Based on the activity schedule defined in Table 6 in the appendix, following secondary power requirements over the mission can be obtained, see Fig. 13 on the left. The galleys are the biggest electrical load $(40.5 \mathrm{~kW})$ and switchedoff at the end of the cruise phase. The hydraulic power for the landing gear actuation is approximately $33 \mathrm{~kW}$, see also Eq. 18. The averaged electrical power distribution for each phase is displayed in Fig. 13 on the right.
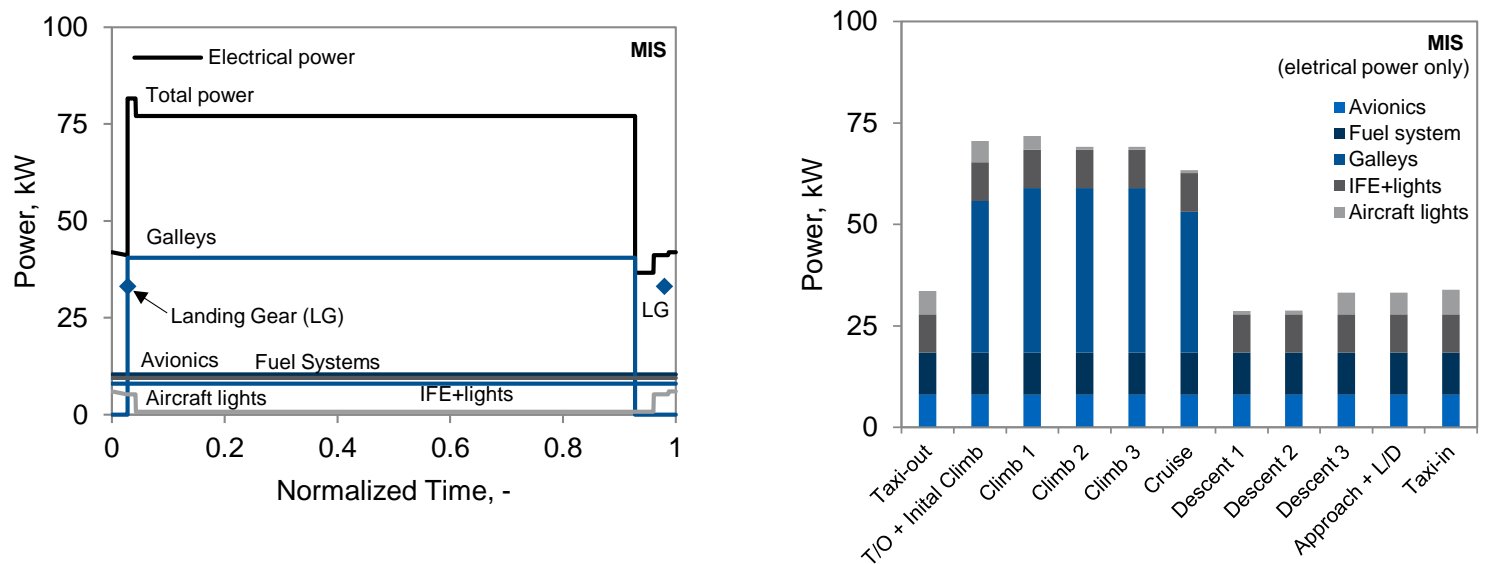

Figure 13. Electrical power requirements of the Miscellaneous Systems (MIS). 


\section{F. Overall Secondary Power Requirements}

Finally, the averaged secondary power requirements of each system in form of electrical, hydraulic and pneumatic (bleed air) power can be summed up, see Fig. 14. It can be recognized, that the electrical power requirements even for the conventional baseline aircraft is very high with approximately $100 \mathrm{~kW}$. Furthermore, the secondary power requirements for the IPS, especially the EIPS lead to high bleed air requirements for the conventional baseline aircraft.

Furthermore, high electrical power requirements can be recognized during climb and cruise phases, mainly due to the galleys. The bleed air requirements depend on the icing envelopes considered and thus the activation of the IPS. For this case study, high bleed air requirements due to the WIPS and EIPS are shown during climb 1, 2 and descent 2 phases. The results of the more-electric baseline aircraft show increases of electrical power requirements in each phase, due to the high power requirements of the CACs of the electrical ECS. Therefore, the bleed air demand can be reduced, due to the no-bleed architecture, since the EIPS is the only system supplied by engine bleed air.
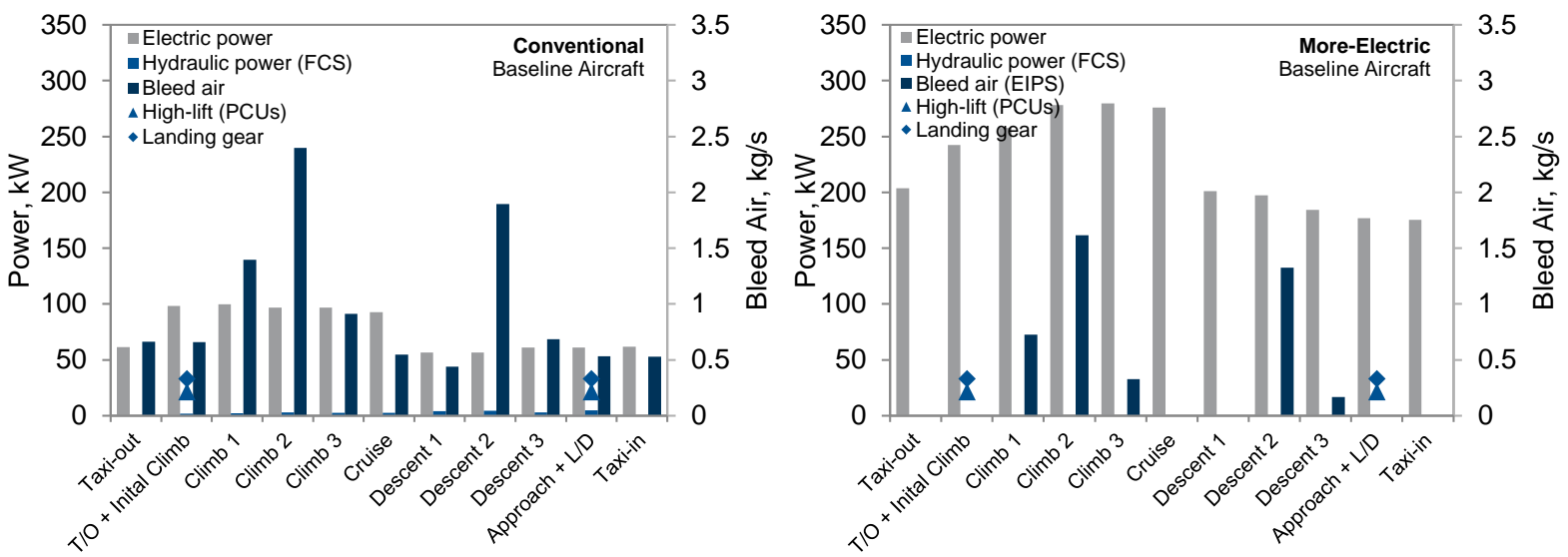

Figure 14. Averaged power requirements of the conventional (left) and more-electric (right) baseline aircraft.

\section{Conclusion}

This study contributes to the integration and assessment of aircraft systems and systems technologies of commercial transport aircraft in preliminary aircraft design. Therefore, a parameterized mission and two representative baseline aircraft, Medium-Range (MR) and Long-Range (LR) aircraft, are defined. Furthermore, to meet the requirements in early transport aircraft design phases, simplified systems model for the main power consuming aircraft systems the Environmental Control System (ECS), the Ice Protection System (IPS) and the Flight Control System (FCS) - are developed. Other remaining and relevant systems power requirements are estimated with data from literature review. The presented approach is integrated into a MATLAB ${ }^{\circledR}$ tool with implemented mission and system objects.

For both, the ECS and IPS, conventional systems ("bleed-system") and more-electric systems ("no-bleed") are considered. The conventional ECS is supplied by the engine bleed air systems, whereas electrical ECS is supplied by ram air and additional electrical Cabin Air Compressors (CAC) to provide the air for cabin air conditioning and cabin pressurization. To protect sensible areas from ice formation or ice accumulations such as wing leading edges, engine inlets (cowling), air data probes and cockpit windows, an IPS for de-icing or anti-icing is necessary. In general, the conventional IPS is supplied by the engine bleed air system to provide the Wing Ice Protection System (WIPS) and the Engine Ice Protection System (EIPS) with hot air. Whereas, for the electrical IPS, the WIPS is realized with attached heating mats inside the leading edge, supplied by the electrical power systems. In this study, the FCSs of the baseline aircraft consist of conventional flight control devices - ailerons, elevators, rudders and spoilers - and of conventional high-lift systems with leading and trailing edge devices at the wings. Two types of actuators, the Servocontrolled Hydraulic Actuator (SHA) and the Electro-Hydrostatic Actuator (EHA) are considered to derive a hydraulic (SHA) and an electrical (EHA) FCS actuation system architecture.

The conducted case study with conventional and more-electric baseline aircraft (MR) show good results compared to published data of similar transport aircraft. Especially the results of the ECS and IPS - which have a sophisticated system model, compared to the other system models - look very valid. The results of the FCS actuation system must be considered carefully, as the system models are very simplified or based on empirical data from literature. Nevertheless, the presented parameterized flight mission enables the aircraft-level assessment of conventional or new aircraft systems and systems technologies in early phases of aircraft design, regarding secondary power requirements. 


\section{Appendix}

Table 3. Mission altitude and speed data for the baseline aircraft (MR/LR).

\begin{tabular}{llcccc}
\hline $\begin{array}{l}\text { Flight Phase } \\
\text { Segment }\end{array}$ & $\begin{array}{c}\text { Altitude, ft } \\
\text { MR/LR }\end{array}$ & MR & $\begin{array}{c}\text { Speed, kt, - } \\
\text { LR }\end{array}$ & Climb Rate, ft/min \\
LR
\end{tabular}

Table 4. Heat flow rate calculations of the Environmental Control System (ECS).

\begin{tabular}{lll}
\hline Heat flow & Equations & Notes \\
\hline Metabolism & $\dot{Q}_{\text {met }}=\dot{Q}_{P A X} \cdot n_{P A X}+\dot{Q}_{\text {crew }} \cdot n_{\text {crew }}$ & $\begin{array}{l}n_{P A X} \quad \text { Number of passengers } \\
n_{P A X} \quad \text { Number of crew members }\end{array}$ \\
Sun (solar) & $\dot{Q}_{\text {sun }}=\dot{q}_{\text {sun }} \cdot A_{\text {window }} \cdot n_{\text {window }} \cdot f_{\text {area }}$ & $f_{\text {area }}=0.5$, window area factor \\
Systems & $\dot{Q}_{\text {sys }}=\dot{Q}_{\text {galleys }}+\dot{Q}_{I F E}+\dot{Q}_{\text {lights }}$ & \\
Conduction & $\dot{Q}_{\text {con }}=\frac{1}{R_{\text {th }}} \cdot\left(T_{\text {skin }}-T_{\text {cabin }}\right)$ & $R_{\text {th }}$ Thermal resistance of the fuselage. \\
\hline
\end{tabular}

Table 5. Heat flux density calculations of the Ice Protection System (IPS).

\begin{tabular}{|c|c|c|c|}
\hline Heat flux & $\begin{array}{l}\text { Equations } \\
\text { (based on Meier and Scholz }{ }^{20}, \text { Chakraborty }^{23} \text { ) }\end{array}$ & Notes & \\
\hline & $\dot{q}_{\text {conv }}=h_{\text {surface }} \cdot\left(T_{\text {surface }}-T_{\text {aw }}\right)$ & & \\
\hline Convection & $h_{\text {surface }}=N u \frac{\lambda}{l_{\text {ref }}}$ & $h_{\text {surface }}$ & Heat transfer coefficient \\
\hline Sensible heating & $\begin{array}{l}\dot{q}_{\text {sens }}=\dot{m}_{\text {local }} \cdot\left\{\left(T_{\text {surface }}-T_{a w}\right) \cdot\left[(1-n) \cdot c_{w}+n \cdot c_{i}\right]+n \cdot L_{f}\right\} \\
\dot{m}_{\text {local }}=V_{\text {TAS }} \cdot \rho_{L W C} \cdot E_{m}\end{array}$ & $\begin{array}{l}\dot{m}_{\text {local }} \\
E_{m}\end{array}$ & $\begin{array}{l}\text { local liquid water mass flow } \\
\text { Water collection efficiency }\end{array}$ \\
\hline Evaporation & $\begin{array}{l}\dot{q}_{\text {evap }}=\dot{m}_{\text {evap }} \cdot L_{e} \\
\dot{m}_{\text {evap }}=0.7 \cdot h_{0} \cdot \frac{R H \cdot e_{w, \infty}-e_{w, \text { surface }}}{\rho_{\infty} \cdot c_{p}} \\
e_{w}=6.1094 \cdot e^{\frac{17.265 T}{243.04+T}}\end{array}$ & $\begin{array}{l}\dot{m}_{\text {evap }} \\
e_{w}\end{array}$ & $\begin{array}{l}\text { Evaporativ mass flow } \\
\text { Saturation vapor pressure }\end{array}$ \\
\hline Kinetic heating & $\dot{q}_{\text {kin }}=\dot{m}_{\text {local }} \cdot \frac{V_{T A S}^{2}}{2}$ & & \\
\hline
\end{tabular}

Table 6. Assumed power requirements of Miscellaneous Systems (MIS).

\begin{tabular}{|c|c|c|c|}
\hline System & $\begin{array}{c}\text { Power Assumptions } \\
\left(\text { based on Steinke }{ }^{17}, \text { Chakraborty }^{23}\right) \\
\end{array}$ & Unit & Activity Schedule \\
\hline Avionics & 8000 & $W$ & All phases \\
\hline Fuel system & 0.136 & $W / \mathrm{kg}$ & All phases (Reference mass: MTOM) \\
\hline Galley & 250 & $W /$ pax & Initial Climb, Climb 1-3, Cruise \\
\hline Cabin lights & 40 & $W / m$ & Al phases (Reference length: Cabin length) \\
\hline In-flight entertainment & 50 & $W /$ pax & All phases \\
\hline \multicolumn{4}{|l|}{ Aircraft lights } \\
\hline Position & 500 & $W$ & All phases \\
\hline Navigation & 50 & $W$ & All phases \\
\hline Beacon & 200 & $W$ & All phases \\
\hline Taxi & 750 & $W$ & Taxi \\
\hline Landing & 4000 & $W$ & Taxi, Take-off, Initial Climb, Approach, Landing \\
\hline Logo & 500 & $W$ & Take-off, Initial Climb, Approach, Landing \\
\hline
\end{tabular}

15

American Institute of Aeronautics and Astronautics 


\section{References}

${ }^{1}$ Tagge, G. E., Irish, L. A., and Bailey, A. R., "Systems Study for an Integrated Digital/Electric Aircraft (IDEA)," NASA Contractor Report NASA-CR-3840, Januar 1985.

${ }^{2}$ Hoffman, A. C., Hansen, I. G., Beach, R. F., Plencner, R. M., Dengler, R. P., et al., "Advanced Secondary Power System for Transport Aircraft," NASA Technical Paper NASA-TP-2463, May 1985.

${ }^{3}$ Sinnet, M., “787 No-Bleed Systems: Saving Fuel and Enhancing Operational Efficiencies,” AERO, Vol. 9, No. 28, 2007, pp. 7-11.

${ }^{4}$ Van Den Bossche, Dominique, "The A380 Flight Control Electrohydrostatic Actuators, Achievements and Lessons Learnt," International Congress of the Aeronautical Sciences ICAS 2006-7.4.1, September 2006.

${ }^{5}$ Lammering, T., "Integration of Aircraft Systems into Conceptual Design Synthesis," PhD Thesis, Institute of Aeronautics and Astronautics, Aachen, May 2014.

${ }^{6}$ Lampl, T., Sauterleute, D., and Hornung, M., “A Functional-Driven Design Approach for Advanced Flight Control Systems of Commercial Transport Aircraft," Proceedings of the 6th International Workshop on Aircraft System Technologies. February 21-22, 2017, Hamburg, Germany, $1^{\text {st }}$ ed., edited by O. von Estorff and F. Thielecke, Shaker, Herzogenrath, 2017, pp. 3-12.

${ }^{7}$ Gillet, S., Nuic, A., and Mouillet, V., "Enhancement in realism of ATC simulations by improving aircraft behaviour models," 2010 IEEE/AIAA 29th Digital Avionics Systems Conference (DASC), 2.D.4-1-2.D.4-13.

${ }^{8}$ Simsic, C. J., "Electric actuation system duty cycles," NAECON '91. National aerospace and electronics conference: Papers, IEEE, 1991, pp. 540-545.

${ }^{9}$ Nuic, A., "User Manual for the Base of Aircraft Data (BADA) - Revision 3.6," July 2004.

${ }^{10}$ Eurocontrol, “Aircraft Performance Database," URL: https://contentzone.eurocontrol.int/aircraftperformance [cited 2 August 2016].

${ }^{11}$ Rustenburg, J. W., Skinn, D. A., and Tipps, D. O., "Statistical Loads Data for the Airbus A-320 Aircraft in Commercial Operations,” DOT/FAA/AR-02/35, April 2002.

${ }^{12}$ Tipps, D. O., Skinn, D. A., Rustenburg, J. W., Jones, T., and Harris, D. A., "Statistical Loads Data for the Boeing 777-200 Aircraft in Commercial Operations," DOT/FAA/AR-06/11, November 2006.

${ }^{13}$ Allen, J. M., “Continuous Descent Final Approach,” URL: https://www.faa.gov/documentLibrary/media/Advisory_Circular/AC_120-108.pdf [cited 11 April 2017].

${ }^{14}$ Wild, T. W., Transport category aircraft systems, IAP, Inc, Casper, WY, 1990.

${ }^{15}$ Moir, I., and Seabridge, A. G., Aircraft systems. Mechanical, electrical, and avionics subsystems integration, $3^{\text {rd }}$ ed., Wiley, Chichester, West Sussex, England, Hoboken, NJ, 2008.

${ }^{16}$ Chakraborty, I., "Subsystem Architecture Sizing and Analysis for Aircraft Conceptual Design," PhD Thesis, Daniel Guggenheim School of Aerospace Engineering, Atlanta, December 2015.

${ }^{17}$ Steinke, T., "Entwicklung einer Methodik zur Modellierung und Analyse von Systemstrukturen im Flugzeugvorentwurf," Diploma Thesis, Institut fuer Luft- und Raumfahrt, Aachen, Germany, November 2010.

${ }^{18}$ Sherif, S. A., Pasumarthi, N., and Bartlett, C. S., "A semi-empirical model for heat transfer and ice accretion on aircraft wings in supercooled clouds," Cold Regions Science and Technology, Vol. 26, No. 3, 1997, pp. 165-179.

${ }^{19}$ Chakraborty, I., Mavris, D. N., Emeneth, M., and Schneegans, A., "An integrated approach to vehicle and subsystem sizing and analysis for novel subsystem architectures," Proceedings of the Institution of Mechanical Engineers, Part G: Journal of Aerospace Engineering, Vol. 230, No. 3, 2016, pp. 496-514.

${ }^{20}$ Meier, O., and Scholz, D., "A Handbook Method for the Estimation of Power Requirements for Electrical De-Icing Systems," Deutscher Luft- und Raumfahrtkongress, September 2010.

${ }^{21}$ Jeck, R. K., "Icing Design Envelopes (14 CFR Parts 25 and 29, Appendix C) Converted to a Distance-Based Format," DOT/FAA/AR-00/30, April 2002.

${ }^{22}$ Roskam, J., “Airplane Design - Part IV: Layout Design of Landing Gear and Systems,” 1989.

${ }^{23}$ Chakraborty, I., Mavris, D. N., Emeneth, M., and Schneegans, A., "A methodology for vehicle and mission level comparison of More Electric Aircraft subsystem solutions: Application to the flight control actuation system," Proceedings of the Institution of Mechanical Engineers, Part G: Journal of Aerospace Engineering, Vol. 229, No. 6, 2015, pp. 1088-1102. 